\title{
Tools to detect structures in dynamical systems using Jet Transport
}

\author{
Daniel Pérez-Palau · Josep J. Masdemont · Gerard \\ Gómez
}

Received: date / Accepted: date

\begin{abstract}
This paper is devoted to the development of some dynamical indicators that allow the determination of regions and structures that separate different dynamic regimes in autonomous and non-autonomous dynamical systems. The underlying idea is closely related to the Lagrangian Coherent Structures concept introduced by Haller. In the present paper, instead of using the Cauchy-Green tensor, that determines the domains where the flow associated to a differential equation is expanding in the normal direction, the Jet Transport methodology is used. This is a semi-numerical tool, that has as basic ingredients a polynomial algebra package and a numerical integration method, allowing, at each integration step, the propagation under a flow of a neighbourhood $U$ instead of a single initial condition. The output of the procedure is a polynomial in several variables that represents the image of $U$ up to a selected order, containing high order terms of the variational equations. Using these high order representation, the places where the normal direction expands can be easily detected, in a similar manner as the procedures for calculating the Lagrangian Coherent Structures do. In order to illustrate the methodology, first the results obtained in the determination of the separatrices of the simple and the periodically perturbed pendulum are given. Later, the applications to the circular restricted three body problem are considered, where the aim is the detection of invariant manifolds of libration point orbits, as well as in the non-autonomous vector field defined by the elliptic restricted three body problem.
\end{abstract}

Keywords Lagrangian Coherent Structures · Jet Transport · Invariant Structures $\cdot$ Simple pendulum · Perturbed pendulum · Circular Restricted Three Body Problem · Elliptic Restricted Three Body Problem

Daniel Pérez-Palau

Departament de Matemàtica Aplicada i Anàlisi, Universitat de Barcelona, Gran Via 585, 08007 Barcelona, Spain

Josep J. Masdemont

IEEC and Departament de Matemàtica Aplicada I, ETSEIB, Universitat Politècnica de Catalunya, Diagonal 647, 08028 Barcelona, Spain

Gerard Gómez

IEEC and Departament de Matemàtica Aplicada i Anàlisi, Universitat de Barcelona, Gran Via 585, 08007

Barcelona, Spain 


\section{Introduction}

The main purpose of this paper is to find different ways to detect hyperbolic structures in dynamical systems. It is known that the hyperbolic structures in autonomous systems (the invariant manifolds) play an important role in the determination of regions of the phase space with different dynamical behaviours. However, their computation is not straightforward in the non-autonomous case. In this paper some indicators are introduced to detect such hyperbolic structures. The base idea is inspired in the Lagrangian Coherent Structures (LCS).

The LCS were introduced by G. Haller [6] at the beginning of the first 2000 decade, and have been used in the study of models appearing in different disciplines. For instance in fluid dynamics $[10,14,16]$ or astrodynamics $[17,4]$. The main use of the LCS is to determine and distinguish different regions of the phase space as a function of the behaviour of the orbits inside each one.

The LCS play the role of the invariant manifolds in non-autonomous systems and should coincide with them in the autonomous case. There are several examples where this comparison has been done. In [7] Haller et al. analysed some cases giving false positives and false negatives in the detection of the invariant manifolds using LCS and, to overcome this drawback, they introduced the so called hyperbolic LCS.

With both tools, LCS and hyperbolic LCS, the detection of the separatrices is done by looking to what happens to neighbouring points in a certain region; if all future trajectories behave in a similar way, then there is no separatrix in that region, if, instead, there are states with different dynamical behaviours, then one can suspect that there exist some object separating them.

To detect such separations Haller et al. proposed to use the Cauchy-Green strain tensor (the transpose of the state transition matrix times itself) and its eigenvalues. The approach used in this paper is different: given a neighbourhood of a given point, the most contractive and expanding directions of the flow, inside the neighbourhood, are determined using the Jet Transport; with this methodology the required computations can be done in a fast and accurate way.

The Jet Transport, also known as flow expansion or box propagation, is a seminumerical tool that allows obtaining the image under the flow map of a given neighbourhood $U$ of a point $x_{0}$. One of the key points is that, evaluating a polynomial, the image under the flow of points inside $U$ can be computed very fast. This technique, introduced by M. Berz and K. Makino to study particle accelerators, has been used in several fields, for instance: to study the close approaches of Near Earth Asteroids [3,2] or to compute a Gaussian particle filter for spacecraft navigation [21].

There are several Jet Transport/Differential Algebra implementations: COSY INFINITY, developed by M. Berz and K. Makino [11], TIDES, developed by Abad et.al. [1] or the CAPD DynSys Library developed by D. Wilczak[22]. For this work the implementation used has been developed by the authors.

The paper is organised as follows: Section 2, introduces the notation and the basic concepts used, mainly the Finite Time Lyapunov Exponents and the LCS, as well as the Jet Transport procedure. Also in this section, a brief explanation of the dynamical models that will be used through the paper is given, together with some reduction and representation tools. Section 3 introduces alternative ways to the computation of LCS and FTLE, that are based on the determination of "maximum initial boxes" and the maximum contraction and expansion directions. Each of the alternatives is analysed and compared with the 
others for four dynamical systems: the simple and the perturbed pendulum and the circular and the elliptic restricted three body problem (CR3BP, ER3BP). The paper ends with a study of practical stability regions in the $\mathrm{CR} 3 \mathrm{BP}$ using the procedures introduced.

\section{Basic definitions and test dynamical models}

In this section, some of the basic concepts and techniques used in the paper are introduced: the Lagrangian Coherent Structures, that give an approximate skeleton of the dynamics of the system, and the Jet Transport, that allows the propagation under the flow of a neighbourhood of an initial condition.

\subsection{The Lagrangian Coherent Structures}

The first definition of the Lagrangian Coherent Structures was given in [6] by means of Finite Time Lyapunov Exponents (FTLE). The underlying idea was to look at the FTLE scalar-field. At each point, $\mathbf{x}$, of the phase space, the associated FTLE is an indicator of the behaviour of the orbits of the neighbouring points to $x$ over a time interval $\left[t_{0}, t_{0}+T\right]$ that measures the average separation between trajectories. A low FTLE value at $\mathbf{x}$ reveals that the orbits of two neighbouring points of $\mathbf{x}\left(x_{1}(0)\right.$ and $\left.x_{2}(0)\right)$ will be close to each other during that time-interval, while a high FTLE value indicates that the orbits of the neighbouring points will have different behaviours, usually because the orbits will diverge from each other. Figure 1 shows an intuitive idea of what kind of orbits we can expect for high and low FTLE.

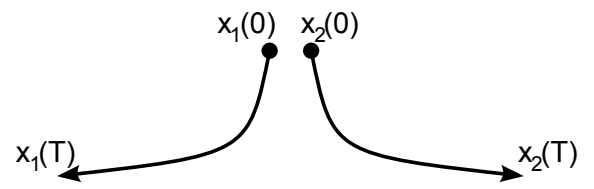

High FTLE

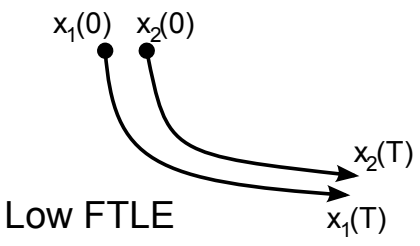

Fig. 1 Qualitative representation of trajectories for different FTLE values.

The FTLE are computed using the flow map. Given a dynamical system represented by the ordinary differential equation,

$$
\dot{\mathbf{x}}(t)=f(\mathbf{x}(t), t)
$$

consider the flow map, denoted by $\phi\left(t ; t_{0}, \mathbf{x}_{0}\right)$ (or $\phi_{t_{0}}^{t}\left(\mathbf{x}_{0}\right)$ whenever the initial and final time are fixed), which is the function that for an initial condition $\mathbf{x}_{0} \in \mathscr{D} \subset \mathbb{R}^{n}$ at time $t_{0}$, gives the state associated to this initial condition at time $t$ :

$$
\begin{aligned}
\phi: \mathbb{R} \times \mathbb{R} \times \mathscr{D} & \longrightarrow \mathscr{D} \\
\left(t, t_{0}, \mathbf{x}_{0}\right) & \longmapsto \phi\left(t ; t_{0}, \mathbf{x}_{0}\right) .
\end{aligned}
$$


Then, the value of the FTLE at $x$ from $t=t_{0}$ up to $t=t_{0}+T$ is defined as:

$$
\sigma_{t_{0}}^{T}(\mathbf{x}):=\frac{1}{|T|} \ln \left\|\frac{\partial \phi_{t_{0}}^{t_{0}+T}(\mathbf{x})}{\partial \mathbf{x}}\right\|_{2}
$$

This is, the FTLE is the normalised square of the largest eigenvalue of:

$$
\left(\frac{\partial \phi_{t_{0}}^{t_{0}+T}(\mathbf{x})}{\partial \mathbf{x}}\right)^{*} \cdot\left(\frac{\partial \phi_{t_{0}}^{t_{0}+T}(\mathbf{x})}{\partial x}\right) \equiv\left(D \phi_{t_{0}}^{t_{0}+T}(\mathbf{x})\right)^{*} \cdot\left(D \phi_{t_{0}}^{t_{0}+T}(\mathbf{x})\right),
$$

where the star indicates transposition. A linear analysis shows that the eigenvector associated to the largest eigenvalue of $\left(D \phi_{t_{0}}^{t_{0}+T}(\mathbf{x})\right)^{*} \cdot\left(D \phi_{t_{0}}^{t_{0}+T}(\mathbf{x})\right)$, which is usually called the CauchyGreen tensor, gives the maximum expansion direction in a neighbourhood of $\mathbf{x}$.

Differences in the propagation behaviour of two close initial conditions can be due to a separating invariant manifold. Hence, the FTLE can be used as indicators of these kind of structures. Unfortunately, hyperbolic invariant manifolds are not the only dynamical structures separating regions. Furthermore, there are some manifolds that are not detected by the LCS method. Therefore, with this first definition it is possible to find false positives and false negatives hyperbolic structures, as is shown in [7].

Figure 2 shows the FTLE scalar-field for the simple pendulum, the perturbed pendulum, the CR3BP and the ER3BP. There one can see how the invariant manifolds appear as the ridges (yellow zones) of the FTLE field. But there also are other ridges that do not correspond to any invariant manifold.

To deal with this situation, G. Haller introduced in [7] the so called Hyperbolic LCS (HLCS). The main idea is to compute, using the linearised flow, the normal repulsion rate $\rho_{t_{0}}^{t}\left(\mathbf{x}_{0}, n_{0}\right)$ and the repulsion ratio $v_{t_{0}}^{t}\left(\mathbf{x}_{0}, n_{0}\right)$. Both elements together measure the ratio of expansion of a normal vector with respect a vector tangent to the structure, that is required for the determination of the HLCS.

Formally, the HLCS are defined as follows: First, consider a material surface $\mathscr{M}(t) \subset \mathscr{D}$ that is defined as a smooth manifold $\mathscr{M}\left(t_{0}\right) \subset \mathscr{D}$ at $t=t_{0}$ advected by the flow into a timeevolving material manifold. A material surface will be an HLCS if it is repelling backward or forward in time.

To measure the repulsion, a unit normal vector $n_{0}$ to $\mathscr{M}\left(t_{0}\right)$ is used. This vector is propagated using the linearised flow given by $D \phi_{t_{0}}^{t_{0}+t}\left(\mathbf{x}_{0}\right)$, and projected to the normal space to obtain the normal repulsion rate of $\mathscr{M}(t)$ :

$$
\rho_{t_{0}}^{t}\left(\mathbf{x}_{0}, n_{0}\right)=\left\langle n_{t}, D \phi_{t_{0}}^{t_{0}+t}\left(\mathbf{x}_{0}\right) n_{0}\right\rangle,
$$

where $n_{t}$ is the unit normal vector to $\mathscr{M}(t)$ at $\mathbf{x}_{t}=\phi_{t_{0}}^{t_{0}+t}\left(\mathbf{x}_{0}\right)$. Observe that if $\rho$ is larger than one, then $\mathscr{M}(t)$ is repelling, while in the contrary, the manifold $\mathscr{M}(t)$ behaves as attracting in the time interval considered.

The objective of the repulsion ratio is to measure the normal expansion in front of the tangential one, so it is defined as:

$$
v_{t_{0}}^{t}\left(x_{0}, n_{0}\right)=\min _{e_{0} \in T_{\mathbf{x}_{0}} \mathscr{M}\left(t_{0}\right)} \frac{\left\langle n_{t}, D \phi_{t_{0}}^{t_{0}+t}\left(\mathbf{x}_{0}\right) n_{0}\right\rangle}{\left\|D \phi_{t_{0}}^{t_{0}+t}\left(\mathbf{x}_{0}\right) e_{0}\right\|},
$$

where $T_{\mathbf{x}_{0}} \mathscr{M}\left(t_{0}\right)$ denotes the set of unit tangent vectors to $\mathscr{M}\left(t_{0}\right)$ at $\mathbf{x}_{0}$.

With the above definitions, $\mathscr{M}(t)$ is said to be normally hyperbolic over $\left[t_{0}, t_{0}+T\right]$ if there exists constants $a, b>0$ such that for all $\mathbf{x}_{0} \in \mathscr{M}\left(t_{0}\right)$ and $\mathbf{n}_{0} \in N_{x_{0}} \mathscr{M}\left(t_{0}\right)$ the following conditions are satisfied: 

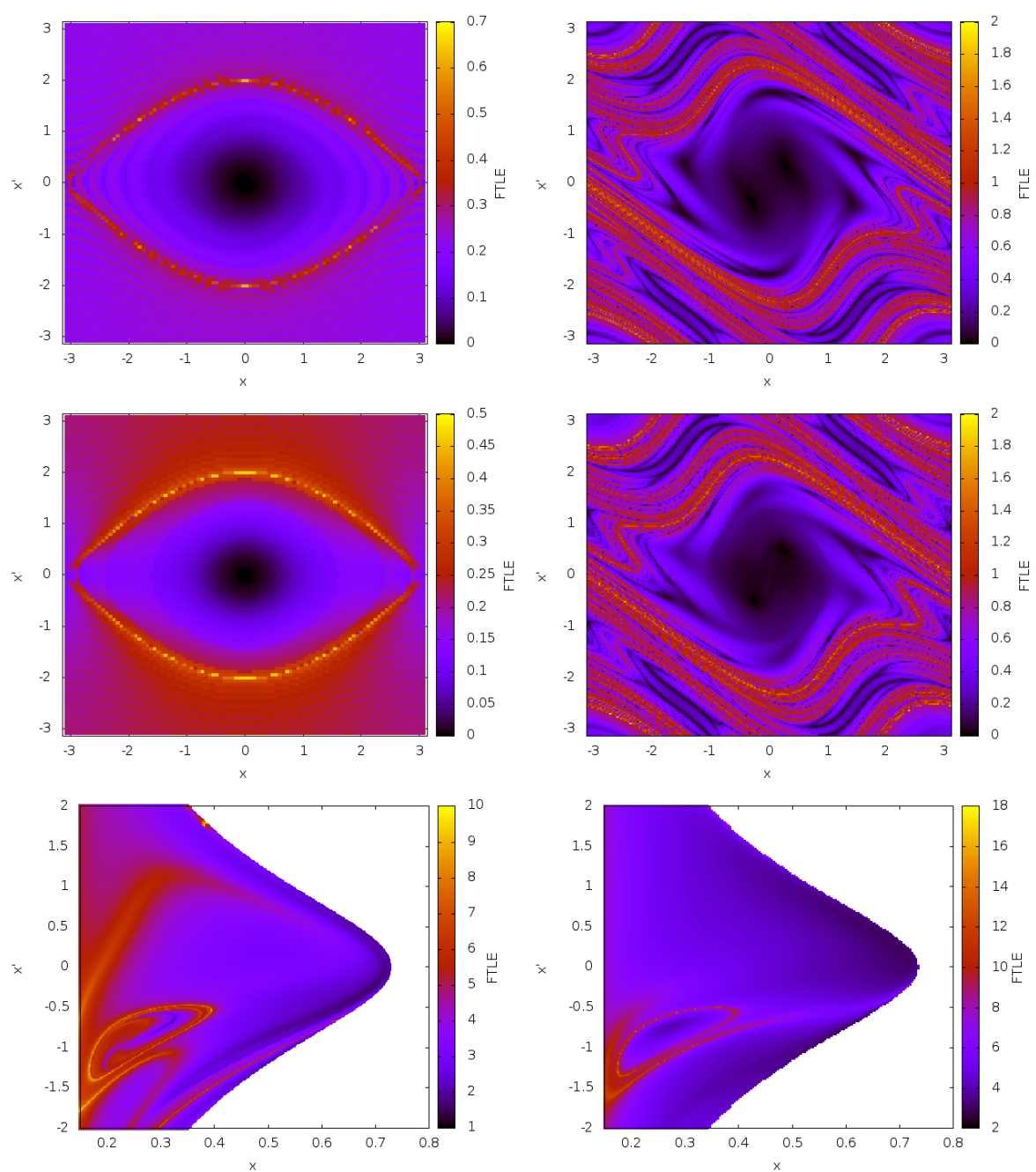

Fig. 2 Finite time Lyapunov exponents scalar field for different systems and values of the computation parameters. From left to right and top to bottom (four top figures): simple pendulum and perturbed pendulum with fixed integration time $T=30$ and $T=10$ respectively; simple pendulum and perturbed pendulum with a close final condition time around $T=30$ and $T=10$ respectively (see section 2.4 for further details about the close final condition time). The two bottom figures correspond to the circular and elliptic R3BP for a fixed final time $T=2$ adimensional units. The mass parameter is $\mu=0.1$, and in the elliptic case $e=0.04$, $f_{0}=0$. The value of the energy used to reduce the dimension of the problem is $E_{0}=\tilde{E}_{0}=-1.725$, and the intersecting condition is given by the hyperplane $y=0$. In the two bottom figures, the grid of points used for the representation is: $\left(x_{i}, 0, \dot{x}_{j}, \dot{y}\left(x_{i}, 0, \dot{x}_{j}, E_{0}\right)\right)$. 
$-\rho_{t_{0}}^{t_{0}+T}\left(\mathbf{x}_{0}, \mathbf{n}_{0}\right) \geq e^{a T}$

$-v_{t_{0}}^{t_{0}+T}\left(\mathbf{x}_{0}, \mathbf{n}_{0}\right) \geq e^{b T}$

Therefore, intuitively, a point belongs to an HLCS if the expansion in the normal direction is larger than the expansion in any tangential direction. This naive idea is in the basis of the alternative ways that will be introduced to compute LCS. Instead of using the FTLE, one can use the ratio at which a vector is expanded and/or contracted.

\subsection{The Jet Transport}

The Jet Transport is a tool that allows the computation of the image, under the flow map, of a set of points around an initial state $\mathbf{x}_{0}$. In contrast, the usual numerical integration methods give only the image of the state $\mathbf{x}_{0}$. To obtain this map, Jet Transport techniques use any numerical integration method for ordinary differential equations (ODE) to obtain the solutions of the variational equations associated to the ODE up to a high order, without writing them explicitly. The direct computation of high order variational equations requires a large amount of work just to obtain the system of differential equations that must be integrated, this is avoided when the Jet Transport is used.

To briefly describe the methodology, consider the system $\dot{\mathbf{x}}=f(x, t)$ and the associated flow map $\phi\left(t ; t_{0}, \mathbf{x}_{0}\right)=x_{t}$ which gives the position at time $t$ of a particle that at time $t_{0}$ is at $\mathbf{x}_{0}$. The objective is now the propagation of a full neighbourhood $U$ of $\mathbf{x}_{0}$, i.e. to compute $\phi\left(t ; t_{0}, U\right)$. For this purpose, the initial condition $\mathbf{x}_{0}$ is replaced by a polynomial that parameterises its neighbourhood: $P_{t_{0}, \mathbf{x}_{0}}(\xi)=x_{0}+\xi=x_{0}+\left(\xi_{1}, \ldots, \xi_{n}\right)^{T}$, where $\xi=\left(\xi_{1}, \ldots, \xi_{n}\right)$ represents the deviation with respect to $\mathbf{x}_{0}$.

Now, any ordinary differential equation integrator (Runge-Kutta, Taylor, symplectic,...) can be used to propagate $\mathbf{x}_{0}+\xi$. Note that, since the initial condition is a polynomial, all the computations required by the integration method have to be done using polynomial algebra, i.e. the algebraic operations are done using truncated polynomials up to a certain degree. In this way one gets $P_{t, \mathbf{x}_{0}}(\xi)$, the expression of the neighbourhood $U$ propagated up to time $t$ by means of truncated Taylor's series up to order $n$. These series give the image under the flow at time $t$ of the initial states $x_{0}+\xi$, i.e. $P_{t, x_{0}}(\xi)=\phi\left(t ; t_{0}, \mathbf{x}_{0}+\xi\right)$. To illustrate this fact, Figure 3 shows a scheme of the basic idea. The left circle represents a neighbourhood $U$ parametrised by $\xi_{1}$ and $\xi_{2}$. The image of this neighbourhood at time $T$, is represented by the ellipse of the right hand side of the plot. The point $P_{T, \mathbf{x}_{0}}(\xi)=$ $\left.\left.\mathbf{x}_{T}+\left(P_{T, \mathbf{x}_{0}}(\xi)\right]_{1}, P_{T, x_{0}}(\xi)\right]_{2}\right)$ is the image of the initial point $\mathbf{x}_{0}+\left(\xi_{1}, \xi_{2}\right)$.

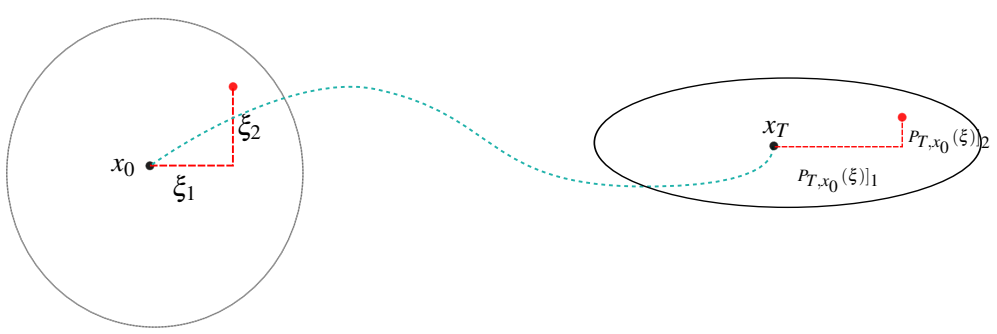

Fig. 3 Scheme of the basic idea underlying the Jet Transport. 
As an illustration of how the Jet Transport works, consider a simple example using a linear center $f(\mathbf{x})=(y,-x)$ and the simplest integration method: the well known Euler's method. According to this method, at each iteration step the system evolves as:

$$
\mathbf{x}_{n+1}=\mathbf{x}_{n}+h f\left(\mathbf{x}_{n}\right) .
$$

Now, instead of using the initial condition $\mathbf{x}_{0}=\left(x_{0}, y_{0}\right)$, take the polynomial,

$$
P_{0, \mathbf{x}_{0}}\left(\xi_{1}, \xi_{2}\right)=\left(x_{0}+\xi_{1}, y_{0}+\xi_{2}\right)
$$

which represents the initial condition plus some perturbation in its neighbourhood.

After a first step of Euler's method, recalling that all the computations must be done with polynomials instead of real numbers, one gets:

$$
\mathbf{x}_{1}=P_{h, \mathbf{x}_{0}}(\xi)=P_{0, \mathbf{x}_{0}}(\xi)+h f\left(P_{0, \mathbf{x}_{0}}(\xi)\right)=\left(\begin{array}{l}
x_{0}+h y_{0} \\
y_{0}-h x_{0}
\end{array}\right)+\underbrace{\left(\begin{array}{cc}
1 & h \\
-h & 1
\end{array}\right)}_{M\left(t_{0}+h\right)}\left(\begin{array}{l}
\xi_{1} \\
\xi_{2}
\end{array}\right)
$$

Note that the independent term of the polynomial $P_{h, \mathbf{x}_{0}}(\xi)$, with respect to the variable $\xi$, corresponds to a first step of the usual Euler method without using the Jet. On the other hand, the matrix $M\left(t_{0}+h\right)$ contains the solution, using Euler's method, of the first variational equations. It should also be noted that there are no higher order terms, because the solution of the second order variational equations is 0 . So, what has been obtained is, $\phi\left(0 ; h, \mathbf{x}_{0}+\xi\right)$.

We can iterate again the procedure to obtain the results for a second Euler's step:

$$
P_{2 h, \mathbf{x}_{0}}(\xi)=\left(\begin{array}{l}
x_{0}+2 h y_{0}-h^{2} x_{0} \\
y_{0}-2 h x_{0}-h^{2} y_{0}
\end{array}\right)+\left(\begin{array}{cc}
1-h^{2} & 2 h \\
2 h & 1-h^{2}
\end{array}\right)\left(\begin{array}{l}
\xi_{1} \\
\xi_{2}
\end{array}\right) .
$$

Again, the two terms obtained correspond to the image of the initial condition $x_{0}$ after this second step (the independent term), and the solution of the first order variational equations for the degree one terms.

Although Euler's method has been used in the above example, any other numerical integration algorithm would work. For instance, using a Runge-Kutta-4 the results are:

$$
P_{h, x_{0}}(\xi)=\left(\begin{array}{cc}
1-\frac{1}{3} h^{2}+\frac{1}{24} h^{4} & \frac{2}{3} h-\frac{1}{12} h^{3} \\
-\frac{2}{3} h+\frac{1}{12} h^{3} & 1-\frac{1}{3} h^{2}+\frac{1}{24} h^{4}
\end{array}\right)\left(\begin{array}{l}
x_{0} \\
y_{0}
\end{array}\right)+\underbrace{\left(\begin{array}{cc}
1-\frac{1}{3} h^{2}+\frac{1}{24} h^{4} & \frac{2}{3} h-\frac{1}{12} h^{3} \\
-\frac{2}{3} h+\frac{1}{12} h^{3} & 1-\frac{1}{3} h^{2}+\frac{1}{24} h^{4}
\end{array}\right)}_{M\left(t_{0}+h\right)}\left(\begin{array}{l}
\xi_{1} \\
\xi_{2}
\end{array}\right) .
$$

Therefore, the only tools needed for the Jet Transport method are: a numerical integration procedure and a polynomial algebra package, that are going to be briefly explained in what follows.

The developed polynomial algebra package includes all the basic operations: addition, product, division, composition of polynomials, and has all the basic functions needed for the implementation of the numerical integration method, as well as some elementary functions (trigonometric, exponential, logarithm, ...). Additionally, some special functions, like the inversion of a function and an equation solver, have also been included.

The second tool needed for the Jet Transport is a numerical integration method for ordinary differential equations. Some results using Euler's method and a Runge-Kutta-4 method have already been shown, however, any other method can be used in a Jet Transport implementation. Higher order Runge-Kutta methods or symplectic integrators, like a leap-frog 
integrator, can be interesting for some applications and can also be adapted to the Jet; the only thing that must be taken into account is the conversion of the standard floating point arithmetics of the numerical integration procedure to a polynomial arithmetic. The integration method used for this paper is the Taylor integration method, as implemented by Jorba and Zou[9] (that must not be confused with the Taylor expansion of the solution with respect to the initial state). The combination of the Jet Transport with a Taylor integration method is called Jet-Taylor method in the following.

Observe that in the Taylor method, at each step the solution can be written in powers of the step-size $h$ as

$$
\phi\left(t_{n}+h ; \mathbf{x}_{n}, t_{n}\right)=\sum_{i=0}^{n} \mathbf{x}^{(i)}\left(\mathbf{x}_{n}, t_{n}\right) h^{i},
$$

and an optimal step, given by

$$
h_{\text {opt }}=\min \left\{\left(\frac{\varepsilon e^{2}\left\|\mathbf{x}^{(1)}\right\|_{\infty}}{\left\|\mathbf{x}^{(N-1)}\right\|_{\infty}}\right)^{\frac{1}{N-2}},\left(\frac{\varepsilon\left\|\mathbf{x}^{(1)}\right\|_{\infty}}{\left\|\mathbf{x}^{(N)}\right\|_{\infty}}\right)^{\frac{1}{N-1}}\right\},
$$

can be used, depending on the precision $\varepsilon$ required (see [18] for more details).

Note that, using this step control, what is controlled is the amount of information that the last two terms of the Taylor expansion in time are giving to the final result, and the control step requirement is that the contribution of these last two terms has to be smaller than a certain amount $\varepsilon$. In addition, it uses relative errors instead of absolute errors, since in some situations the values of the variables can be large, specially when dealing with high orders.

For the Jet Transport with $l$ variables, each $x_{m}^{(i)}$ is a polynomial $x_{m}^{(i)}=\sum_{k} c_{m, k}^{(i)} \xi^{k}$ (where $m$ stands for the index of the variable). Therefore, the control step should be done for every coefficient of the polynomial. Using

$$
\psi_{k}^{i}=\max _{1 \leq m \leq l}\left|c_{m, k}^{(i)}\right|
$$

the optimal step becomes,

$$
h_{o p t}=\min _{k}\left\{\left(\frac{\varepsilon e^{2} \psi_{k}^{1}}{\psi_{k}^{N-1}}\right)^{\frac{1}{N-2}},\left(\frac{\varepsilon \psi_{k}^{1}}{\psi_{k}^{N}}\right)^{\frac{1}{N-1}}\right\} .
$$

In this way all the orders of the jet are controlled.

Finally, a comment about the size of the $U$ neighbourhoods (boxes) that can be propagated. As for the step-size control, there is also a domain, that can be fixed, where the propagation obtained by the jet transport has errors below a given tolerance. The problem is how to estimate this domain. In analogy with the step-size control selection, some estimations on the box-size can be done using the higher order terms on the final jet. Assuming that, at the end of the integration, the jet can be written as

$$
\phi\left(t ; \mathbf{x}_{0}+\xi, t_{0}\right) \approx P_{t_{0}+t, \mathbf{x}_{0}}(\xi)=\sum_{|k| \leq n} \mathbf{a}_{k} \xi^{k}
$$

with $\mathbf{a}_{k}=\left(a_{1, k}, \ldots, a_{l, k}\right)$ the coefficients of the Taylor approximation of the $m$-th variable with multi-index exponent $k$, then, a straightforward option is to select the size of the box in 
such way that the last term does not add more than a certain tolerance, $\varepsilon_{\text {jet }}$, to the series, i.e: $a_{m, k} \xi^{k} \leq \varepsilon_{\text {jet }}$.

Assuming that the box has to be of the same size in any direction, one gets

$$
\xi_{\max }=\min _{\substack{|k|=n \\ 1 \leq m \leq l}}\left(\frac{\varepsilon_{\mathrm{jet}}}{\mathbf{a}_{\mathbf{m}, \mathbf{k}}}\right)^{\mathbf{1} / \mathbf{k}} .
$$

Observe that if the points in a certain neighbourhood have very different behaviours, then the value of $\xi_{\max }$ will be small. On the other hand, if all of them behave similarly, then it will be large. Therefore $\xi_{\max }$ is going to be a good indicator to determine objects that separate the dynamics.

The above procedure suffers from a drawback when the last term of the resulting propagated series is zero. Consider, for instance, the series of the scalar function $\sin (x)$, around $x=0$, truncated at order 4 . In this case, the jet is

$$
P(\xi)=\xi+0 \cdot \xi^{2}-\frac{1}{6} \cdot \xi^{3}+0 \cdot \xi^{4}
$$

and, according to the above criteria, the maximum radius is unbounded. Unfortunately, there are no a priory analytical error estimations to overcome these kind of situations. In these cases, is better to study the decayment of the non-zero coefficients of the polynomial and use an exponential fit of the size of all the known non-zero coefficients up to order $n$ to obtain an accurate estimate of the size of the $n+1$ order (see also [23]). This criterion can also be used as a general criterion and not only when the previous one fails.

\subsection{Dynamical test models}

The first two dynamical models that will be used are the simple pendulum and a timeperiodic perturbation of it:

$$
\ddot{x}=-\sin (x), \quad \ddot{x}=(2.5 \cos 5 t-1) \sin (x) .
$$

As it is well known, the pendulum has two main differentiated regimes: circulations and librations. Between them there are two special orbits connecting the unstable fixed point and separating both regimes. These two orbits are the invariant manifolds of the unstable point, and are the kind of hyperbolic structures to be detect. In the perturbed pendulum, the hyperbolic structures change with time and become more complex; they are associated to the invariant manifolds of the periodic orbit that substitutes the unstable equilibrium point.

The other two examples considered through the paper are the circular and the elliptic restricted three body problems. The CR3BP studies the motion of a massless particle, for instance a spacecraft or an asteroid, under the gravitation force of two massive bodies (primaries) moving in circular orbits around their center of mass. Only the planar case will be considered. Using suitable dimensionless coordinates and units, the equations of motion can be written as (see Szebehely [20]):

$$
\begin{aligned}
& \ddot{x}-2 \dot{y}=\Omega_{x}, \\
& \ddot{y}+2 \dot{x}=\Omega_{y},
\end{aligned}
$$


where $\Omega(x, y)=\left(x^{2}+y^{2}\right) / 2+\mu / r_{1}+(1-\mu) / r_{2}+\mu(1-\mu) / 2, r_{1}=\sqrt{(x+1-\mu)^{2}+y^{2}}$, $r_{2}=\sqrt{(x-\mu)^{2}+y^{2}}$, and $\mu$ and $1-\mu$ are the normalised masses of the the small and large primary, respectively. The above equations have a first integral that can be written as

$$
E(x, y, \dot{x}, \dot{y})=\frac{1}{2}\left(\dot{x}^{2}+\dot{y}^{2}\right)-\Omega(x, y) .
$$

The CR3BP (2) has five equilibrium points: three of them are collinear with the primaries and exhibit, for any value of $\mu$, a saddle $\times$ center behaviour. The other two are located in the third vertex of an equilateral triangle formed with the two main bodies and for small values of the mass parameter $\mu$ they are of center $\times$ center type.

Due to the center part of the collinear points, there are periodic orbits around them, the so called Lyapunov periodic orbits, and thanks to the saddle component, these periodic orbits have stable and unstable invariant manifolds. These invariant manifolds are the hyperbolic structures to be detected.

The ER3BP considers the motion of a massless object within the gravitational potential field generated by a two-body system following elliptical orbits around the center of mass. To maintain the position of the primaries fixed in a rotating reference frame, a periodic pulsation is added to the synodic system with the resulting equations:

$$
\begin{aligned}
& x^{\prime \prime}-2 y^{\prime}=\frac{1}{1+e \cos (f)} \Omega_{x}, \\
& y^{\prime \prime}+2 x^{\prime}=\frac{1}{1+e \cos (f)} \Omega_{y},
\end{aligned}
$$

where $e$ is the eccentricity of the ellipse of the primaries and $f$ is the true anomaly, which now substitutes time as independent variable. When the eccentricity is equal to 0 the problem becomes the CR3BP. When $e>0, E$ is no longer a first integral; despite that, one can use a pseudo-energy

$$
\hat{E}(x, y, \dot{x}, \dot{y})=\frac{1}{2}\left(\dot{x}^{2}+\dot{y}^{2}\right)-\frac{\Omega(x, y)}{1+e \cos (f)},
$$

in order to do some reductions of the dimension of the system.

Both restricted three body problems have already been approached using Lagrangian Coherent Structures. For the CR3BP, Tricoche et al. in [17] give some results about how to use this tool. In the elliptic case, Gawlik et al. in [4] have done a systematic study of how the different parameters of the problem (the mass parameter $\mu$, the eccentricity $e$, and the initial true anomaly $f$ ) modify the structure of the LCS obtained.

\subsection{Representation of the results in LCS computations}

The usual representation of LCS is done showing the FTLE scalar field used for its computation, this is: a two-dimensional colored plot such that each point in the plot corresponds to a state (or its 2-dimensional projection) of the phase space, and the color represents the magnitude of the FTLE. In this section it will be shown how to deal with higher dimensions, together with some alternatives to get better representations.

For one, or one and a half, degrees of freedom dynamical systems, like the pendulum or the perturbed pendulum, the full phase space can be represented in a 2-dimensional plot. However, for systems with more degrees of freedom there is no such straightforward option. For two degrees of freedom systems, there are several options to reduce the dimension. 
A usual one is the use a certain surface of section and, in some cases, a first integral of the differential equations. In the CR3BP the hyper-plane defined by $y=0$ together with Jacobi's first integral allows a representation of the phase space (at each energy level) using the $(x, \dot{x})$ plane. In the ER3BP, one can use the pseudo-energy $\hat{E}$ to reduce the dimension. Of course, one can use different hyper-planes as surfaces of section and try to extract from them the desired structure.

The top left plot of Figure 2 shows the FTLE field for the pendulum. One can see that, in addition to the main yellow curve associated to the invariant manifolds of the unstable equilibrium point, there appear some extra (dark) curves over and under the invariant manifolds. To explain the appearance of these curves, recall that all the motions of the pendulum are periodic $(\bmod 2 \pi)$. Then, when the value of $T$ for the computation of the FTLE is fixed, there are points in the phase space that at $t=T$ are again at its initial state (i.e. the points with a period dividing the final time $T$ ) while for other initial conditions the final state is far from the initial one. These last ones are responsible of the false structures. To avoid them to appear, the final time $T$ must be selected as a function of the initial condition in such way that the initial and final states coincide, or, at least, are as close as possible. The second row of Figure 2 has been obtained using this modified final time. Now, all the "false positives" obtained using a fixed final time have disappeared. In return, the resulting plots are no longer continuous, showing discontinuities when the number of revolutions done by the pendulum changes.

The above strategy is not feasible for the perturbed pendulum. In this case not all the orbits are periodic and, therefore, there is no a final time $T$ for which the initial and final states coincide. Instead, one can look for a time $T$ such that in the time interval $[0.8 T, 1.2 T]$ the initial and final states are close enough. For the simple pendulum this procedure gives the same result obtained when a suitable value of $T$ for each initial condition is used, since the distance between the final and initial states is zero, but for the perturbed pendulum there are no major differences when they are compared with the results obtained using a fixed value of $T$.

As in the simple pendulum case, the high FTLE ridges obtained for the periodically perturbed pendulum correspond to invariant manifolds. When the perturbation is added, the hyperbolic fixed point of the simple pendulum becomes a periodic orbit with the period of the perturbation. This periodic orbit has stable and unstable invariant manifolds that are the ones detected by the high values of the FTLE. Furthermore, at the top and bottom part of the image there are high FTLE values corresponding to a part of the invariant manifolds of other two periodic orbits with period twice the one of the perturbation. Finally, there are also low FTLE regions (dark regions) corresponding to periodic orbits. Figure 4 shows two examples of such kind of periodic orbits. The first one is located in one of the top most black zone in the central stable region, the other one is in the bottom right of the stable region. Both initial conditions can be identified in the top plot of Figure 4.

In higher dimensional problems, like the restricted three body problem, there appear also false structures, (see [15]). In the CR3BP some structures correspond to intersections of invariant manifolds, others, which are not invariant manifolds, are associated to periapsis passages with respect to one of the primaries. Recall that, when a particle is at the periapsis it moves faster and, therefore, the neighbouring particles diverge, which causes this appearance of large FTLE values. 

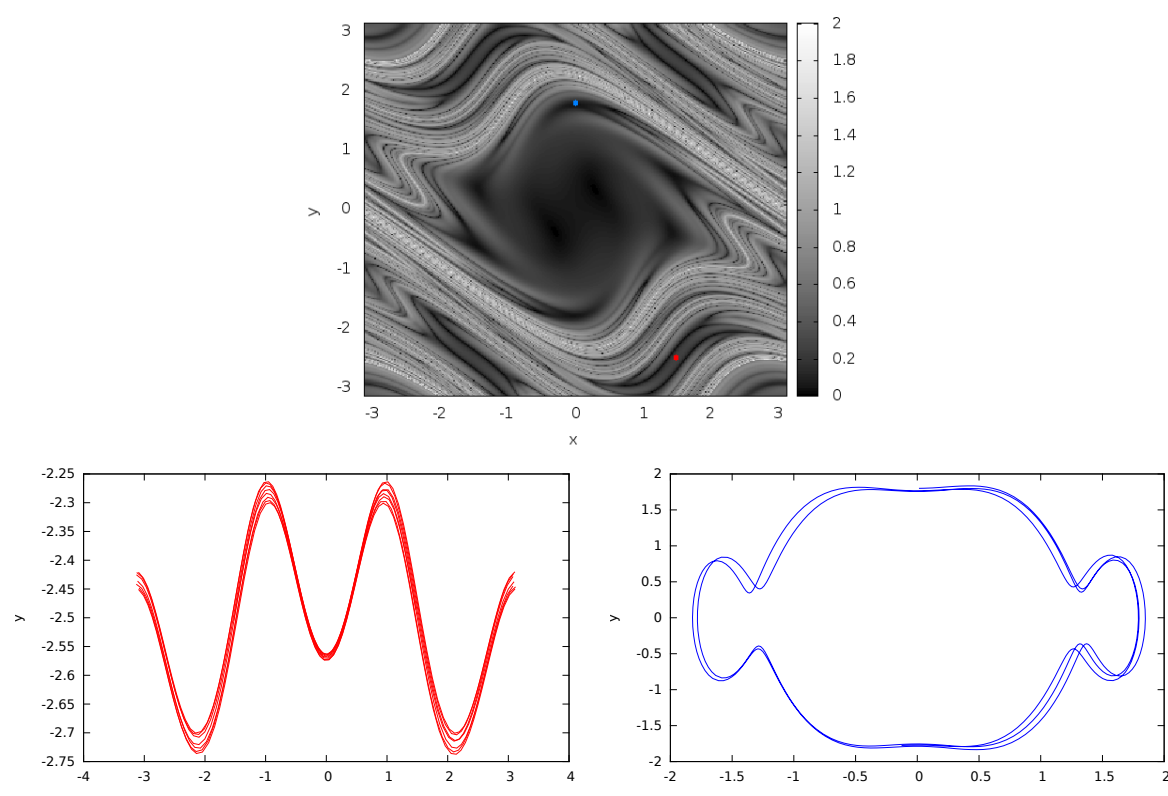

Fig. 4 Two examples of orbits in stable regions close to periodic orbits with period twice the period of the perturbation (left) and six times the period of the perturbation (right). The top figure shows the location of the initial conditions of both orbits (near $(1.5,-2.5)$ and $(0.0,1.5)$ ).

\section{Non-linear alternatives for the computation of LCS}

In this section three different methods for the computation of the LCS are introduced, they make use of the high order variational terms provided by the Jet Transport. The first method uses the maximum initial neighbourhood that can be used by Jet Transport ensuring a certain accuracy. The other two procedures are based on the detection of the maximum expansion and contraction directions and the associated ratios of expansion and contraction.

In all the numerical computations that follow, the order of the Taylor method used for the numerical integration has been set equal to 25 , and the order $n$ of the jet expansions has been taken equal to 5 for the two pendulums and equal to 2 for the restricted three body problems.

In principle, it could be enough to use a jet of order 1 to detect the hyperbolic structures (with less CPU effort) and, in fact, all the algorithms that follow can be used with any order of the jet. However, the procedures that require the determination of expansion and contraction rates, need two consecutive orders of the jet for the computation of the derivatives, so, in these two cases, at least order 2 must be used.

\subsection{Maximal initial boxes}

If a point is close to a hyperbolic structure, after some time the points in its neighbourhood can behave in different ways and, in general, they will diverge from each other. As a result, the final box (image of the initial neighbourhood) will increase its size, making impossible to approximate their positions, within a certain accuracy, using a polynomial of a given degree 
(maybe except if the size of the initial box is reduced enough). On the contrary, if the point is far away enough from a hyperbolic structure, then the surrounding points will behave in a more regular way and it will possible to use bigger initial boxes.
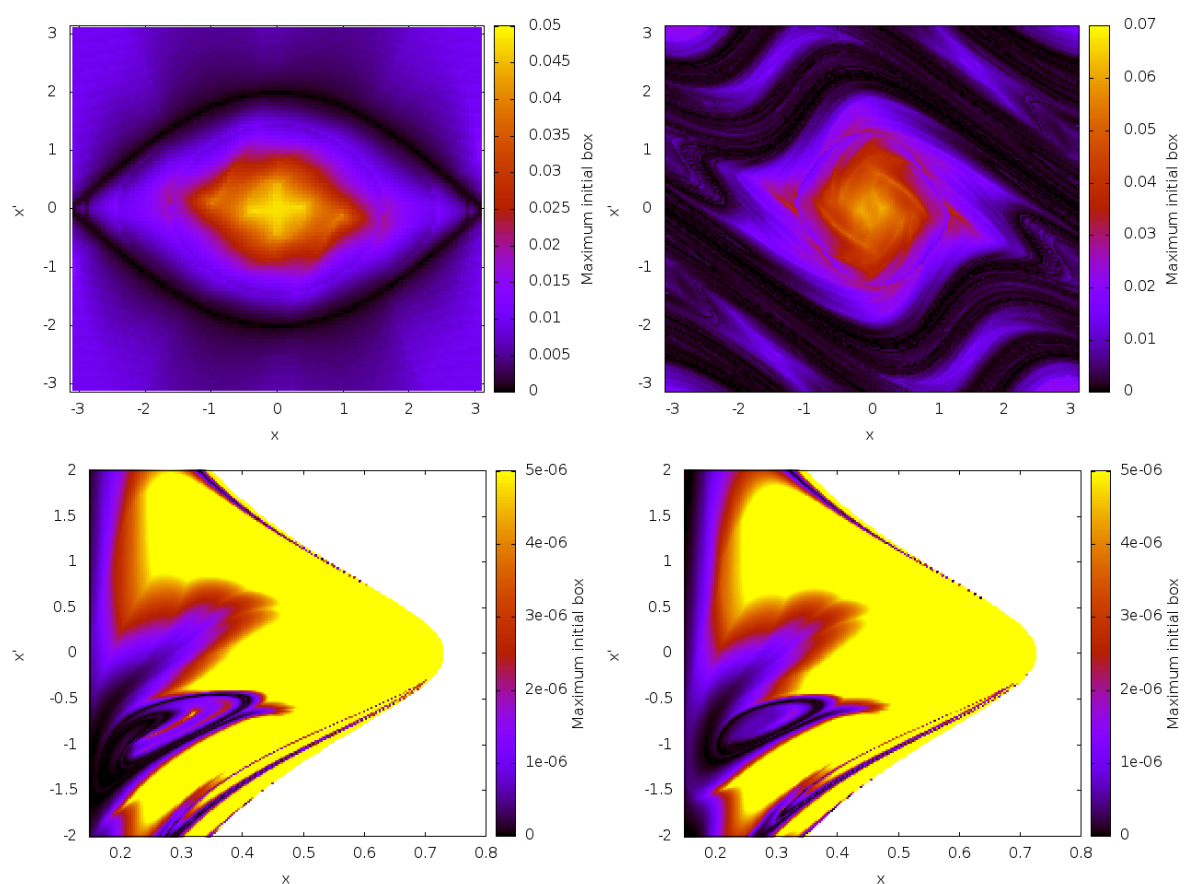

Fig. 5 Size $\xi_{\max }$ of the maximum initial boxes to maintain a given precision $\varepsilon_{j e t}=10^{-6}$ for the simple and perturbed pendulums (top) and for the CR3BP and ER3BP (bottom). In the pendulum case, the computations have been done using a final time around $T=10$. The two bottom figures have been computed with a fixed final time $T=2$ adimensional units. In these two last examples, the mass parameter is $\mu=0.1$, the values of the Jacobi constant used to reduce dimension of the problem are $E_{0}=-1.8$ and $\tilde{E}_{0}=-1.8$ and the hyperplane condition is $y=0$. The initial phase for the elliptic problem is $f_{0}=0$. This means that the grid used for the computation of the initial conditions is of the form: $\left(x_{i}, 0, \dot{x}_{j}, \dot{y}\left(x_{i}, 0, \dot{x}_{j}, E_{0}\right)>0\right)$.

Attending to the above remark, the first indicator considered is the size $\xi_{\max }$ of the maximum initial box that can be taken such that the propagated images fulfil the following $\varepsilon_{\text {jet }}$ precision requirement:

$$
\xi_{\max }=\min _{|k|=n}\left(\frac{\varepsilon_{\mathrm{jet}}}{a_{m, k}}\right)^{1 / k},
$$

where $a_{m, k}$ is the coefficient of the Taylor approximation of the $m$-th component of the solution obtained by the jet $\phi_{m}\left(t ; \mathbf{x}_{0}+\xi, t_{0}\right)=\sum_{|k| \leq n} a_{m, k} \xi^{k}$

Figure 5 shows the maximum size of the initial boxes to maintain a given precision $\varepsilon_{j e t}=10^{-6}$ for a grid of points for the simple pendulum as well as for the CR3BP and the ER3BP. As it can be seen, the points that are close to an invariant manifold have lower values than all those that are far away. The basic structures detected agree well with the ones determined using FTLE and displayed in Figure 2. 
Figure 6 shows the difference between the maximum box sizes, $\xi_{\max }$, computed using the jets of order 1 and 5 . It can be seen that near the hyperbolic structures the improvement for the size of the box is small (of the order of $10^{-5}$ ) while close to the origin the improvement is increased almost up to 0.1 . This means that the use of a high order jet allows the detection of the regular regions with less computational effort.

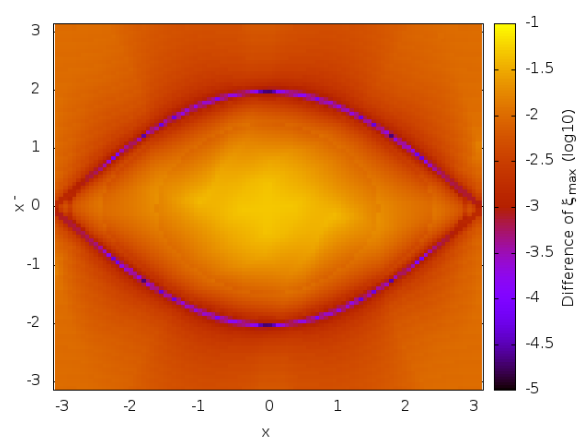

Fig. 6 Difference between the two sizes, $\xi_{\max }$, determined using jets of order 1 and 5 , and a precision $\varepsilon_{\text {jet }}=$ $10^{-6}$, for a pendulum with a final integration condition time close to $T=30$.

\subsection{Expansion and contraction measures}

Using the same basic idea introduced in [7] for the determination of the hyperbolic LCS, one can obtain similar results computing the maximum expansion and maximum contraction ratios from the results given by the Jet Transport. This is what is considered and discussed in this section.

In the 1-degree of freedom case, one can consider a circle of radius $r_{0}$ around an initial condition $x_{0}$ and, on this circle, look for the angle $\theta_{+}$associated to the most expanding direction as well as to the one corresponding to the most contracting one $\theta_{-}$, i.e. the angles such that the difference $\| \phi\left(0 ; T, x_{0}\right)-\phi\left(0 ; T, x_{0}+\xi(\theta) \|\right.$ is maximum and minimum, respectively, and subjected to $\|\xi(\theta)\|=r_{0}$. Once the results of the Jet Transport are available, instead of computing the images of the points on the circle by numerical integration, one can obtain the values of $\phi\left(0 ; T, \mathbf{x}_{0}+\xi(\theta)\right)$ in an easy and fast way. The procedure proceeds as follows:

1. Take an initial state $x_{0}$.

2. Propagate the state together with its jet up to a certain time $T$. In this way the polynomial $P_{T, x_{0}}(\xi)$ approximating $\phi\left(0 ; T, x_{0}+\xi\right)$ is determined.

3. Compute the maximum initial box and define its radius as $r_{0}$.

4. Using $P_{T, x_{0}}(\xi)$, compute the images of a first grid of initial conditions, on the circle of radius $r_{0}$, parametrised by an angle $\theta$ :

$$
\left(x_{0}, \dot{x}_{0}\right)+\xi(\theta)=\left(x_{0}, \dot{x}_{0}\right)+\left(r_{0} \cos \theta, r_{0} \sin \theta\right) .
$$

Determine a first approximation of the angles giving the maximum expansion, $\lambda_{+}=$ $\max _{\theta}\left\|P_{T, x_{0}}(\xi(\theta))-P_{T, x_{0}}(0)\right\|$ and the maximum contraction, $\lambda_{-}=\min _{\theta} \| P_{T, \mathbf{x}_{0}}(\xi(\theta))-$ $P_{T, x_{0}}(0) \|$, with $\|\xi(\theta)\|=r_{0}$. 
5. Use the initial approximations obtained in the previous step to refine the values of $\lambda_{ \pm}$ using a maximum/minimum search algorithm. The computations are done by means of Newton's method together with the results of the Jet for the required derivatives.

6. Compute the expansion and the contraction rates, $\lambda_{ \pm} / r_{0}$.

The results obtained with the above procedure, for the simple and perturbed pendulums, are displayed in Figure 7. Note that, in all the cases, those zones with big expansion or contraction rates coincide with the ones with high FTLE shown in Figure 2, so both the expansion and contraction rates can also be used for the detection of the hyperbolic LCS separating structures. This procedure can be extended to higher dimensional systems. In what follows it is shown how to proceed with both the circular and the elliptic R3BP. A main difficulty is how to represent the resulting data. As it has already been mentioned, this problem is solved by fixing a value of the energy $(E)$ (or the pseudo-energy $(\hat{E})$ ), and fixing an hyperplane $y=0$.
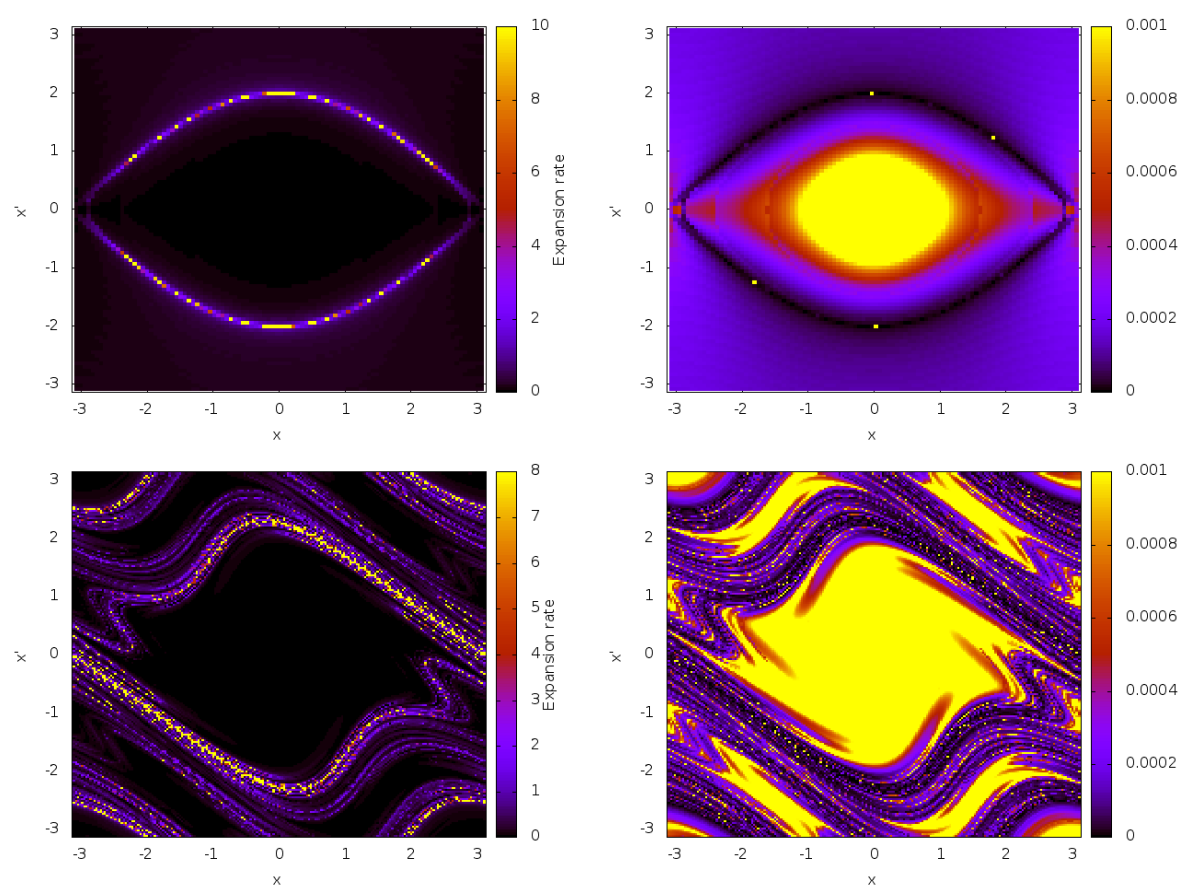

Fig. 7 For the simple pendulum (top) and the perturbed pendulum (bottom), maximum expansion rates (left) and maximum contraction rates (right). In all cases, the rates have been computed using the Jet Transport with a final time close to $T=10$ time units.

In the computation of the contraction and expansion rates, the additional dimensions also affect the way on how the directions of maximum expansion or contraction are determined. There are two possible ways to proceed: the simplest approach is to compute the expansions and contractions only in the directions of the two-dimensional grid used to represent the results; a more accurate procedure would require the exploration of all possible directions in the full phase space. For the first approach, the expansion and contraction directions are computed taking into account neighbourhoods only in the 2D grid directions, i.e. taking a 
circle in $(x, \dot{x})$, parameterised by the angle $\theta$ and fixing $y=0$ and $\dot{y}=\dot{y}\left(x, \dot{x}, E_{0}\right)$. This is, the perturbation $\xi$ to be considered into the polynomial is:

$$
\xi=\left(r_{0} \cos \theta, 0, r_{0} \sin \theta, \dot{y}\left(r_{0} \cos \theta, r_{0} \sin \theta, E_{0}\right)\right) .
$$

Using the angle $\theta$ as parameter, one can apply the procedure in a similar way as in the $2 \mathrm{D}$ case.

For the second approach, the search must be carried out in the full 4D space. Now the computation of the first approximations of the expanding and contracting directions is done using a parameterisation of the 3 -sphere given by three angles $\left(\theta_{1}, \theta_{2}, \theta_{3}\right)$. Once suitable initial seeds are obtained, they are refined using a gradient method in order to get more accurate maximum expansion and contraction direction rates. Figure 8 shows the expansion and contraction rates in the elliptic R3BP using both approaches. In both cases, one can distinguish those zones of high hyperbolicity for its high expansion and contraction rates.
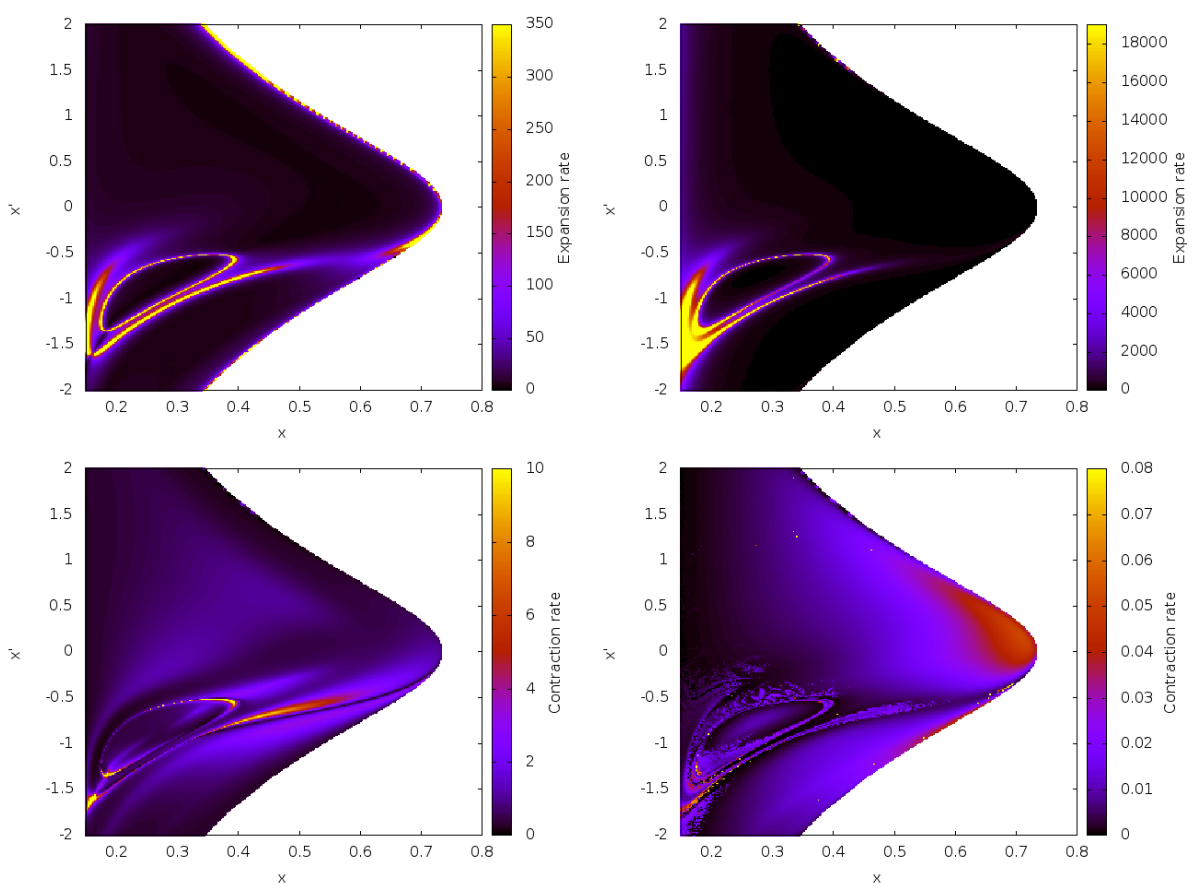

Fig. 8 Expansion (top) and contraction (bottom) rates for the ER3BP with mass parameter $\mu=0.1$ and eccentricity $e=0.04$, taking values in a grid on $(x, y, \dot{x}, \dot{y})=\left(x_{i}, 0, \dot{x} j, \dot{y}\left(x, \dot{x}, \hat{E}_{0}\right)\right)$, with $\hat{E}_{0}=-1.725$. The figures on the left have been obtained using the 2D search, while those on the right doing the search in the full 4D space.

It has been found that the contraction rates are not as good indicators as the expansion rates, essentially for some numerical issues. The main one is related to the slow convergence rate of the gradient method when there are several close angles for which their associated values are also very close to the minimum. In these cases, the method quickly reaches this set of values but it gets stuck for some iterations. These conflictive cases, where the gradient works with problems, usually happen near the invariant structures to be detected. 
In order to speed up the procedure for these ill conditioned cases, some improvements have been implemented. Taking into account that in these cases the iterations of the gradient method almost repeat the same direction search every two iterates, the results are averaged every two iterations getting in this way a better search direction. Also for these cases is a good practice to combine the gradient method with other one-dimensional minimisation procedures, like Newton's method or a golden section search. The performance of these implementations is displayed in Figure 9. When the gradient method is followed by a 1dimensional Newton's method, to determine the minima in the direction of the gradient, a very slow convergence is obtained, as is shown by the blue curve of the figure. When the directions given by the gradient are averaged, the results are much better, although after some iterations (around 400) the difference between two consecutive angles is very small (in fact below the value fixed as one of the stopping criteria of the algorithm). This is shown by the red and green curves of the figure. Nevertheless, if the stop condition is removed, there is a monotonous decrease of the distance function, as is shown by the purple line.
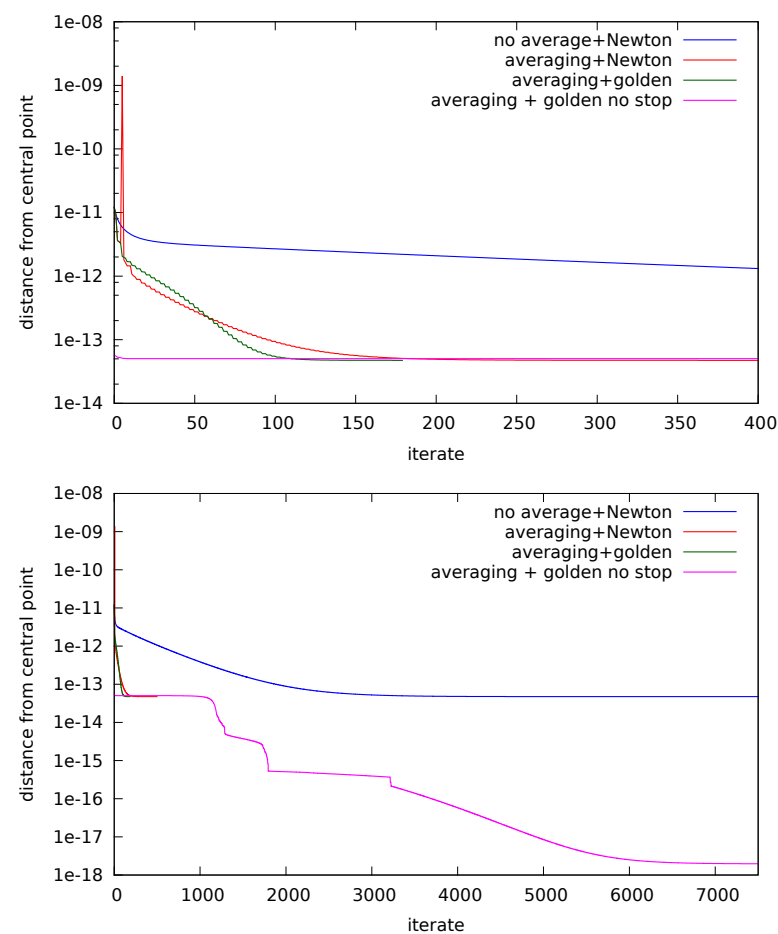

Fig. 9 Evolution (in quadruple precision) of the minimum distance obtained by the gradient method as a function of the number of iterations for different methodology improvements. Blue: in the standard gradient method a 1-dimensional Newton's method is used to determine the minima in the direction of the gradient. Red and green: the decreasing direction is computed as the average of the last two gradient iterations and the determination of the minima along this direction is computed using Newton's method (in red) or a golden section search (in green). The purple line shows the behaviour of the gradient method followed by a golden section search without any stop condition if two consecutive angles are very close. The top plot shows the distance from the center for the first 400 iterates while the bottom one corresponds to the 7500 first iterates. 
In order to see how the LCS evolve when changing one of the parameters of the problem, a visualisation algorithm has been developed that allows the extraction of the desired structure from different data plots. Let us see how to determine the evolution of the central circle-shaped curve, detected in the lower left part of the plots in Figure 8, when the initial true anomaly is changed. The expansion rate has been selected as dynamical indicator, although any other one could be used. Figure 10 shows the obtained results for the ER3BP with a fixed value of eccentricity $e=0.04$, mass parameter $\mu=0.1$ and two different values of the initial phase: $f_{0}=0$ and $f_{0}=\pi / 2$. The initial pseudo-energy $\hat{E}_{0}$ is represented in the $z$-axis. These results are in good agreement with the ones obtained in [4] using a completely different procedure.
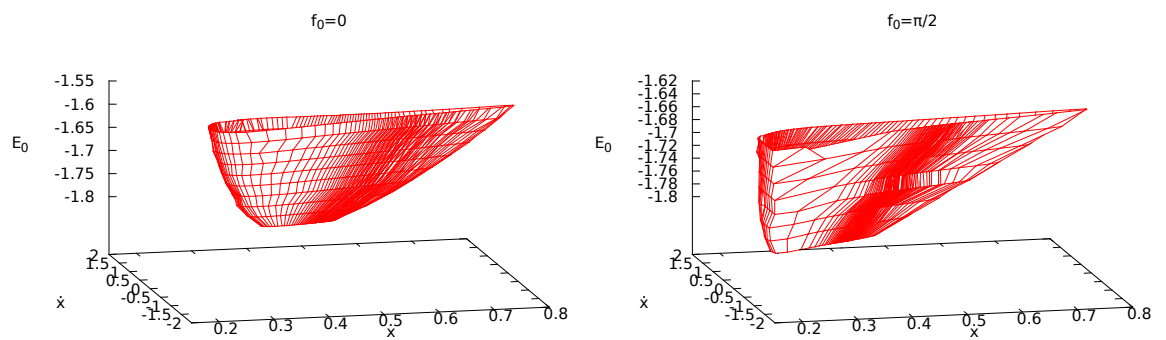

Fig. 10 Intersection of the plane $y=0$ with the LCS corresponding to the time-dependent analogue of the stable manifold of a periodic orbit around the $L_{1}$ point in the ER3BP. The initial true anomaly is $f_{0}=0$ (left) and $f_{0}=\pi / 2$ (right), in both plots the eccentricity is $e=0.04$ and the mass parameter $\mu=0.1$. The structures have been computed using the expansion rate as indicator for two different values of the initial phase. Taking as initial condition $x, \dot{x}$ inside a grid, $y=0$ and $\dot{y}=\dot{y}\left(\hat{E}_{0}\right)$ being the value of $\dot{y}$ such that the energy is equal to $\hat{E}_{0}$. The range of pseudo-energies used moves from $\hat{E}_{0}=-1.8$ to $\hat{E}_{0}=-1.64$ in the first case and from $\hat{E}_{0}=-1.8$ to $\hat{E}_{0}=-1.58$ in the second one.

\section{Detection of practical stability regions}

It is well-known that the triangular equilibrium points of the CR3BP are linearly stable for values of the mass parameter $\mu$ below the Routh's critical value $\mu_{1}$. It is also known that in the spatial case they are nonlinearly stable, not for all the initial conditions in a neighborhood of the equilibrium points $L_{4}, L_{5}$ but for a set of relatively big measure. The fraction of stable motions tends to 1 when the size of the neighborhood tends to 0 , for almost all $\mu \in\left(0, \mu_{1}\right)$. This follows from the celebrated Kolmogorov-Arnold-Moser theorem. In fact, there are neighborhoods of computable size for which one obtains "practical stability", in the sense that the massless particle remains close to the equilibrium point for very long time intervals.

An important question is which part of this stability subsists when the idealised CR3BP is substituted by the Earth-Moon system with its real motion and under the very strong influence of the Sun and the milder perturbations due to planets, solar radiation pressure, no spherical shape of the Earth and Moon, etc. This has been studied since long time ago in [12, $5,19]$. In [19] it is shown how the invariant manifolds of $L_{3}$ enclose the practical stability zones.

These zones of practical stability can be detected using the FTLE as well as any other of the indicators presented in this paper. Here are shown the results obtained when one 
considers initial conditions in a grid of points in the $x-y$ plane with zero initial velocities $(\dot{x}=\dot{y}=0)$.
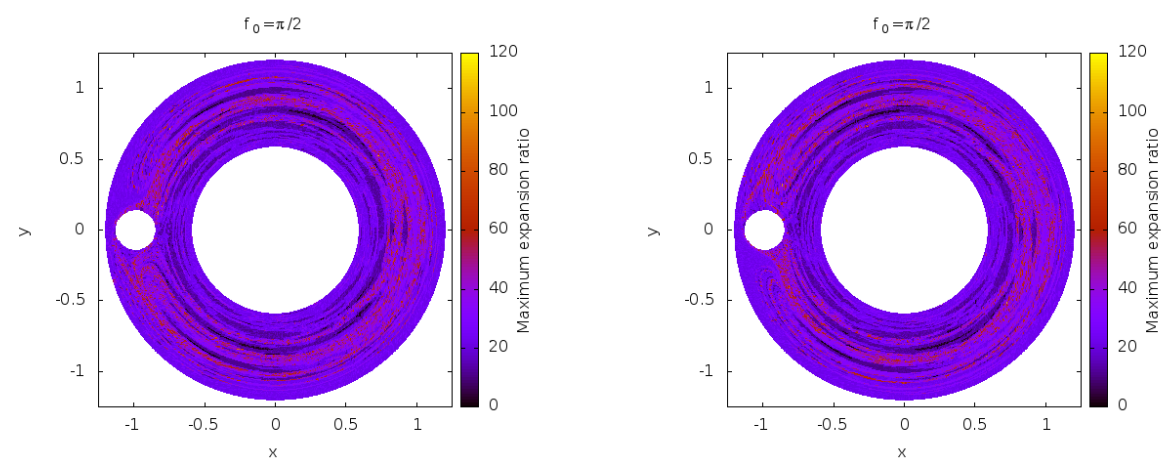

Fig. 11 Maximum expansion for the CR3BP in forward time (left) and backward time (right). There appear stable zones (darker regions) around the big primary and close to $L_{4 / 5}$ points.

The results for the maximum expansions, using the 4D search, are shown in Figure 11 for positive $T=30$ (left) and negative $T=-30$ (right) final times of integration. To avoid extremely long integration time intervals, initial conditions close to the main primaries have been avoided, as well as those far from them, which correspond to stable orbits. In the figures, one can detect the stable zones as those associated to low expansion rates. Note that these zones are not symmetric with respect to the $x$-axis. This asymmetry can be explained because the indicator depends on the integration time interval as well as if the integrations has been done forward or backwards in time. In both figures, one can see two large regions around $L_{4}$ and $L_{5}$, located at $(1 / 2-\mu, \pm \sqrt{3} / 2= \pm 0.866$..), corresponding to the practical stability regions described in the references already mentioned, as well as other stable zones produced by other mechanisms.

Forward time integrations are useful to reveal stable hyperbolic structures while backwards ones to reveal unstable ones. Both hyperbolic structures can be seen in Figure 11.

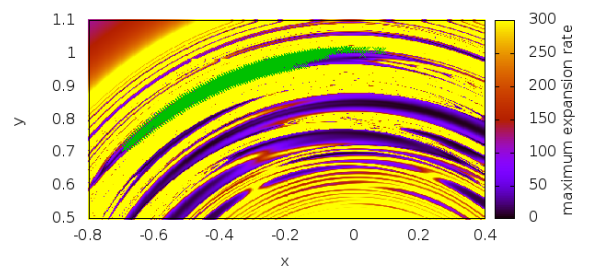

Fig. 12 Zoom around the $L_{5}$ point showing the practical stability region using the maximum expansion rate as indicator. The stability region computed in [5] is also displayed (in green).

Figure 12 shows the a magnification of the stable region around $L_{5}$. The practical stability region computed in [5], using a completely different approach, is also displayed (in green) to show the good agreement between these two completely different procedures. The 
same plot shows other stable regions, some of them are even bigger than the stability zone around $L_{5}$, and are analysed with more detail in what follows.
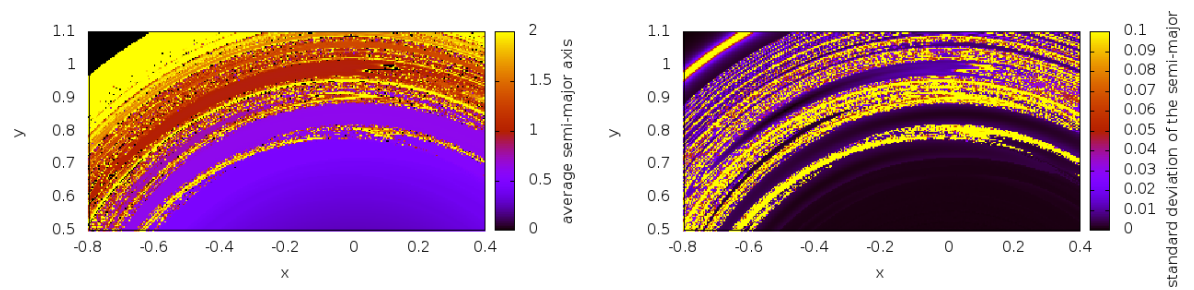

Fig. 13 Average (left) and standard deviation (right) of the semi-major axis of the orbits around the practical stability region considered as Keplerian orbits with respect to the larger primary.

Figure 13 shows the average and the standard deviation of the semi-major axis of all the orbits represented in Figure 14 considered as Keplerian orbits with respect to the larger primary. There one can see how, indeed, the orbits with smaller standard deviations are those ones related to the stable zones while the ones with big deviations are close to the unstable zones. The big deviations are produced when the perturbation of the small mass affects the orbit in a notorious way and, therefore, is far from a Keplerian motion. Therefore, the stable zones are filled with orbits that are never close to the small primary.

In Figure 14 the dark green region corresponds to the already known practical stability zone around $L_{4}$. The motions in the red region are close to almost periodic orbits (precessing Keplerian ellipse) around the large primary, with a basic period close to three times the period of the small primary $(2 \pi)$. Analogously, the motions in the light-green region are close to a almost periodic orbits, with a basic period close to $5 \times 2 \pi$. The ones in the cyan region are again precessing ellipses with a basic period close to $2 \times 2 \pi$. When we approach the unstable region the motion described becomes more irregular, as can be seen in the orbits displayed in Figure 14. The ones in the boundaries are not as close to be periodic as the ones in the center of the stable region. The unstable zone between both regions is filled with orbits that due to the gravitational influence of the small primary, are far from a precessing Keplerian ellipse.

\section{Conclusions}

The Jet Transport is a semi-analytical tool that allows the propagation of a neighborhood of an initial condition by means of high order truncated polynomials. This paper develops a methodology based on the Jet Transport integration to detect structures in dynamical systems. The method is tested with models like the simple pendulum and the restricted three body problem, both circular and elliptical, with great success. For the computation of Lagrangian Coherent Structures, the determination of directions with extreme expansion and contraction rates by means of the Jet Transport give equivalent results as Haller's methodologies, based on the Cauchy-Green tensor computations. Other interesting applications are related with the determination of practical stability regions in dynamical systems that classically have been addressed by means of FTLE or similar indicators. For this item, the CR3BP is used as a test example. The Jet Transport methodology easily identifies the stable regions in a clear way, including some of them that were already reported in the classical literature. 

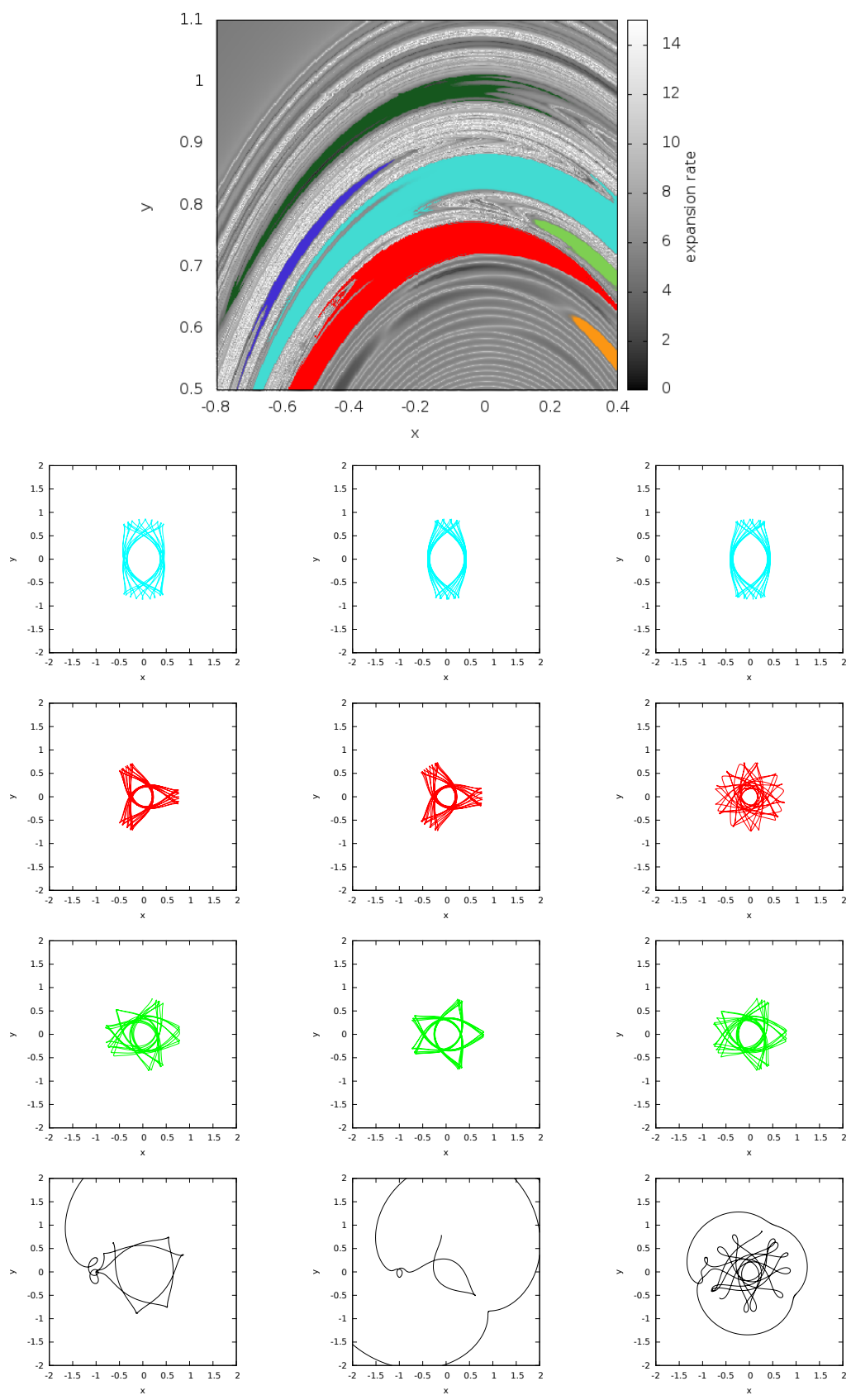

Fig. 14 Top: Maximum expansion rates around $L_{4}$ computed with a final time $T=30$. The colored highlighted regions correspond to stability regions, the dark green on the top is the practical stability region around $L_{4}$. The colored orbits displayed have initial conditions in the region of the same color. The last row corresponds to orbits in the unstable regions. 


\begin{tabular}{|c|c|c|c|c|c|}
\hline$k_{0}$ & $k_{1}$ & power & division & sinus & cosinus \\
\hline 0 & 0 & -14.41550 & -15.8465 & -16.7759 & -16.495 \\
\hline \hline 1 & 0 & -14.98994 & -15.5148 & -15.8057 & -15.8871 \\
\hline 0 & 1 & -15.05401 & -15.5068 & -15.7906 & -15.8517 \\
\hline \hline 2 & 0 & -14.77298 & -15.1239 & -15.137 & -15.1591 \\
\hline 1 & 1 & -14.56881 & -14.9689 & -14.9576 & -14.9978 \\
\hline 0 & 2 & -14.83939 & -15.1173 & -15.1112 & -15.1446 \\
\hline \hline 3 & 0 & -14.61939 & -14.7187 & -14.5815 & -14.5823 \\
\hline 2 & 1 & -14.40447 & -14.356 & -14.3343 & -14.3222 \\
\hline 1 & 2 & -14.38862 & -14.2958 & -14.3417 & -14.3127 \\
\hline 0 & 3 & -14.66474 & -14.6576 & -14.5848 & -14.563 \\
\hline
\end{tabular}

Table 1 Decimal logarithm of the difference of the coefficient associated to the exponents $k_{0}, k_{1}$, computed using the polynomial algebra and Pari/GP for some basic functions: power, quotients, sinus and cosinus. For the computations with Pari/GP multi-precision arithmetic has been used.

As is common in this kind of indicators, the main drawback is the CPU time required for their computation. This can be critical when the grid of points used for the computations is very dense, in order to get very sharp results. On the other hand the indicators are able to capture accurately the non-linear dynamics.

Acknowledgements The authors would like to thank to the anonymous reviewers for their comments and fruitful suggestions to improve the quality of the paper. This work has been supported by the Spanish grants MTM2010-16425, MTM2013-41168-P (G.G.), MTM2012-31714, 2014SGR504 (J.J.M.), and MTM2010-16425, MTM2013-41168-P, AP2010-0268 (D.P.).

\section{A Numerical results for the Jet Transport and the polynomial algebra}

Before proceeding with the detection of dynamical structures, let us first present some of the tests we use to evaluate the efficiency of the implementation of the polynomial algebra package and its application to the Jet Transport.

To perform this comparative we have used Pari/GP [13]. This package implements two relevant things: from one side it supports multi-precision arithmetic. This is very suitable to check the accuracy of our computations, since the solution provided with Pari/GP has very low truncation errors and can be considered a reference. From another side, Pari/GP also includes polynomial algebra computations making the tests even simpler.

Then, as a first test, we have performed the basic algebraic operations using our own algebra package and Pari/GP. Subtracting the resulting polynomials we can see how much the coefficients differ. These tests have been done, for each algebraic operation, 1000 times (using randomly generated polynomials) in order to compute the averaged errors. As we have already mentioned, the accuracy of Pari/GP is higher than the machine precision, therefore the computations done with Pari/GP can be used to validate the ones of the algebra package.

Table 1 shows the decimal logarithm of the error for the power $\left(P^{\alpha}(\xi)\right)$, the division $(P(\xi) / Q(\xi))$, and the trigonometric functions $(\sin (P(\xi)), \cos (P(\xi))$ ), taking polynomials $P(\xi)$ and $Q(\xi)$ with two variables and degree three. It can be seen that the precision of the computations is close to the machine precision.

Two more tests have been done to check the precision of the Jet Transport computations. For these ones we have used the simple pendulum and the CR3BP as reference models. The first one has to do with the accuracy of the derivatives of the flow map obtained by the Jet Transport compared with the values obtained by numerical differentiation using:

$$
f^{\prime}(x) \approx \frac{f(x+\delta)-f(x-\delta)}{2 \delta}
$$

For the pendulum and the CR3BP cases, Figure 15 shows the differences between the first order derivatives, which are related to the first order variational equations, (the five first derivatives for the pendulum and the first order ones for the CRBP) obtained from the Jet Transport and the numerical values of the variational 

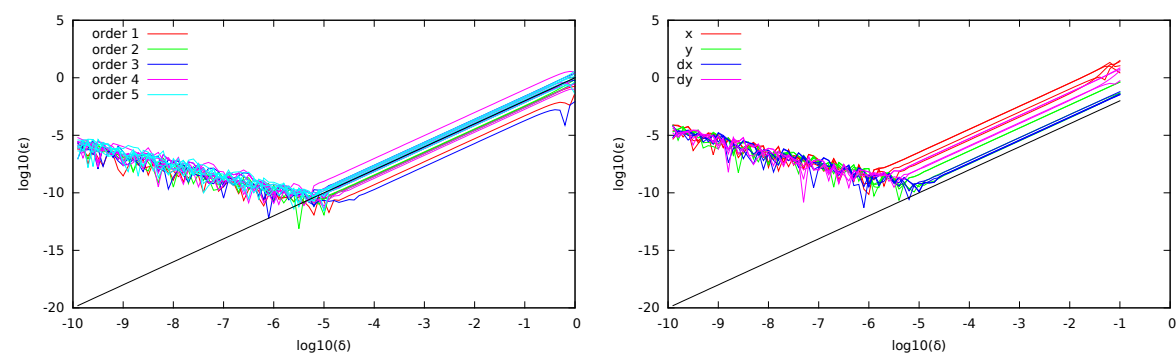

Fig. 15 Accuracy tests for the computation of the variational equations using the Jet Transport. Left: After 200 time units for a pendulum model (with initial conditions $(x, y)=(1,0)$ ), and as a function of $\delta$, values of the difference between the $i$-th order variational equations computed with the Jet Transport and the values obtained using numerical derivatives. For $i=1,2,3,4,5$ (respectively red, green, blue, magenta and cyan). Right: After 100 time units in the CR3BP (with initial conditions $(x, y, \dot{x}, \dot{y})=(0.9,0.3,0.1,0.4)$ ), and as a function of $\delta$, values of the difference between the first order variational equations computed with the Jet Transport and the values obtained using numerical derivatives for each one of the four variables of the system.

equations computed using the previous differentiation formula. As usual with this kind of numerical computations, when we decrease the value of $\delta$ the errors become smaller until a certain value of $\delta$ from which the round-off error in the calculation of the difference becomes larger than the truncation error. To improve this results we implement a Richardson extrapolation applying the recursive formulae:

$$
\begin{aligned}
F_{1}(h) & =f^{\prime}(h), \\
F_{i+1}(h) & =F_{i}(h) \frac{F_{i}(h)-F_{i}(2 h)}{2^{i+1}-1} .
\end{aligned}
$$

Using these formulae we are able to get better approximations of the numerical derivatives. Figure 16 shows the results of five steps of Richardson extrapolation when we consider the first variable $x$ of the CR3BP. From this figure, it is clear that at each extrapolation step the errors decrease and that the approximation given by the jet is very good, the error is of the order of $10^{-13}$ using double precision arithmetic.

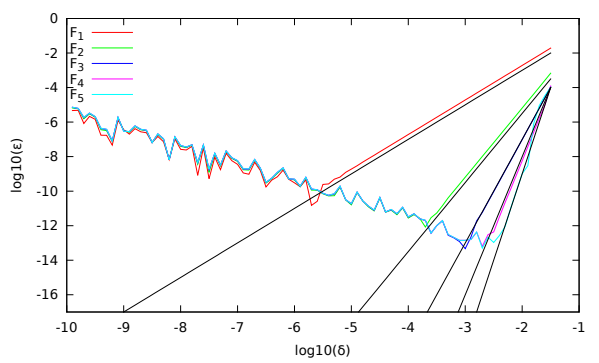

Fig. 16 Accuracy test for the first order variational terms in the CR3BP after 2 time units. The colour curves display the difference between the first order variational equations of the first coordinate $\left(\frac{\left.\partial \phi_{t_{0}}^{T}\left(x_{0}\right)\right]_{1}}{\partial x}\right)$ computed with the Jet Transport and the values obtained using the Richardson extrapolation step $F_{i}$ as a function of $\delta$ in $\log 10$ scale. The black lines show the trends expected according to the order of the formula.

The last test that has been done is related to the conservation of a first integral. Instead of checking its conservation as a scalar value, we check it as a polynomial. First we compute an initial polynomial giving the variations of the values of the first integral associated to small deviations of the variables. Then, at some point during the integration, we can compute again the first polynomial integral and check its components against the initial values. Figure 17 shows the difference between the first, second and third order terms of 
the polynomial first integral along the integrations of the pendulum and the CR3BP differential equations. As expected, the longer the time the bigger the error, but always within a good accuracy.
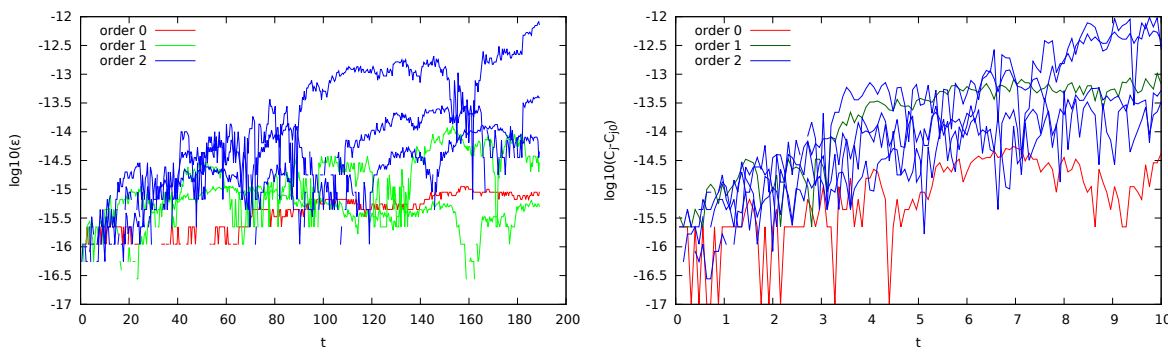

Fig. 17 Accuracy tests for the conservation of the first integral (Left: pendulumwith initial condition $(x, y)=$ $(1,0)$, Right: CR3BP). As a function of time, differences between the initial energy polynomialwith initial conditions $(x, y, \dot{x}, \dot{y})=(0.9,0.3,0.1,0.4)$ and the one computed using the jet for the independent term (in red), the first order terms (in green) and second order terms (in blue).

The above results ensure that it is possible to extract information about the high order variational terms from the output of the Jet Transport computations. The main drawback of the Jet Transport is that, in case that some information is needed for a single point, computations will be slow compared to a single standard integration. However, if we are looking for information of neighbouring points, as is the case in many Monte Carlo simulations, or we need high order variational terms, then the Jet Transport becomes very useful. Figure 18 shows the amount of CPU time needed to integrate different amounts of initial conditions of the CR3BP, during 20 time units, in front of the CPU time required by the Jet Transport to get the images under the flow of the same initial conditions for the same amount of time. We can appreciate the almost constant behaviour of the Jet in front of the linear growth using a RK78 classical procedure.

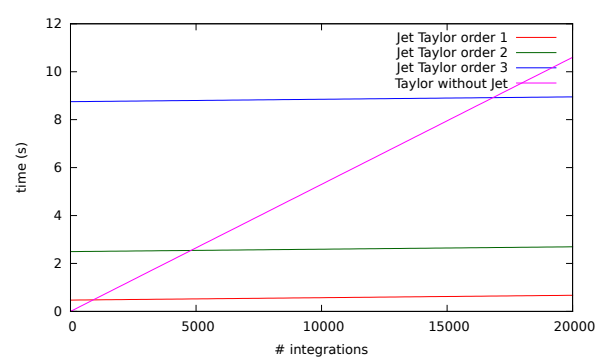

Fig. 18 In the CR3BP, CPU Time in seconds spent as a function of the number of initial conditions propagated using the Jet-Taylor method with orders 1,2,3 (respectively red, green and blue) and a usual Taylor Method without jet propagation (magenta). Each integration is done in a randomly chosen orbit close to the initial condition at $(1,0,0)$ with initial velocity $v_{0}=(1,1,1)$ during 20 adimensional time units. The mass parameter is $\mu=0.012$. The computations have been carried out with an Intel Xeon(R), CPU E5645, 2.40GHz.

\section{References}

1. A. Abad, R. Barrio, F. Blesa, M. Rodríguez: Algorithm924: TIDES, a Taylor Series Integrator for Differential Equations. ACM Trans. Math. Softw. 39(1):1-28 (2012). 
2. R. Armellin, P. Di Lizia, F. Berenlli-Zazzera, M. Berz: Asteroid Close Encounters Characterization Using Differential Algebra: the Case of Apophis. Celestial Mech. and Dynam. Astronom. 107(4):451-470 (2010)

3. E.M. Alessi, A. Farrès, A. Vieiro, À. Jorba, C. Simó: Jet transport and applications to neo's. Proceedings of 1st IAA Planetary Defense Conference ESA Conference Bureau, Granada (Spain), 2009.

4. E. S. Gawlik, J. E. Marsden, P. C. Du Toit, and S. Campagnola: Lagrangian coherent structures in the planar elliptic restricted three-body problem. Celestial Mech. \& Dynam. Astronom., 103(3):227-249 (2009).

5. G. Gómez, À. Jorba, J.J. Masdemont and C. Simó: Dynamics and Mission Design Near Libration Points. Vol. IV Advanced Methods for Triangular Points. World Scientific (2001).

6. G. Haller and G. Yuan: Lagrangian coherent structures and mixing in two-dimensional turbulence. Physica D: Nonlinear Phenomena 147(3-4):352-370 (2000).

7. G. Haller: A variational theory of hyperbolic Lagrangian Coherent Structures. Physica D: Nonlinear Phenomena 40(7):574-598 (2011).

8. À. Jorba: A methodology for the numerical computation of normal forms, centre manifolds and first integrals of Hamiltonian systems. Exp. Math. 8(2):155-195 (1999).

9. À. Jorba, and M. Zou: A software package for the numerical integration of ODE's by means of high-order Taylor methods. Exp. Math., 14(1):99-117 (2005).

10. F. Lekien, N. Leonard: Dynamically consistent Lagrangian coherent structures. Experimental chaos 742,pp 132-139 (2004).

11. K. Makino, M. Berz: COSY INFINITY 9.1 Programmer's Manual. MSU Report MSUHEP101214, Michigan State University, East Lansing, MI. (2011).

12. R. McKenzie and V. Szebehely: Nonlinear stability motion around the triangular libration points. Celestial Mech. \& Dynam. Astronom. 23 (3), 223-229 (1981).

13. PARI/GP, version 2.5.3, Bordeaux (2012), http://pari.math.u-bordeaux.fr/.

14. J. Peng, J. O. Dabiri: Transport of inertial particles by Lagrangian coherent structures: application to predator-prey interaction in jellyfish feeding. Journal of Fluid Mechanics, 623, pp 75-84 (2009), doi:10.1017/S0022112008005089

15. D. Pérez, G. Gómez, J.J. Masdemont: Detecting invariant manifolds using hyperbolic Lagrangian coherent structures. Paper number IAA-AAS-DyCoSS1-08-06, IAA Conference on Dynamics and Control of Space Systems. "Final papers". Porto (2012).

16. S. Shadden, F. Lekien, J. E. Marsden: Definition and properties of Lagrangian coherent structures from finite-time Lyapunov exponents in two-dimensional aperiodic flows. Physica D: Nonlinear Phenomena 212.3, 271-304 (2005).

17. C. Short, K. Howell: Lagrangian coherent structures in various map representations for application to multi-body gravitational regimes. Acta Astronautica Vol 94, Issue 2, February 2014, pp 592-607

18. C. Simó: Global dynamics and fast indicators. Global analysis of dynamical systems 373-389, Inst. Phys., Bristol (2001).

19. C. Simó, P. Sousa-Silva and M. Terra: Practical Stability Domains Near L4,5 in the Restricted ThreeBody Problem: Some Preliminary Facts. Progress and Challenges in Dynamical Systems, Springer Proceedings in Mathematics \& Statistics 54, 367-382 (2013).

20. V. Szebehely: Theory of Orbits: The Restricted Problem of Three Bodies. ,682 pages, Academic Press, London (1967).

21. M. Valli, R. Armellin, P. Di Lizia, M. Lavagna: Nonlinear Filtering Methods for Spacecraft Navigation Based on Differential Algebra. Acta Astronautica, Vol. 94, issue 1, 2014, pp. 363-374

22. D. Wilczak: CAPD DynSys Library documentation. The CAPD group [online], available: mnich.ii.uj.edu.pl/capd/doc4

23. A. Wittig, P. Di Lizia, R. Armellin, K. Makino, F. Bernelli-Zazzera, M. Berz: Propagation of Large Uncertainty Sets in Orbital Dynamics by Automatic Domain Splitting. To appear in Celestial Mech. \& Dynam. Astronom. (2015). 